\title{
An Approximate Dynamic Programming Approach for Coordinated Charging Control At Vehicle-to-Grid Aggregator
}

\author{
Jinbiao Xu and Vincent W.S. Wong \\ Department of Electrical and Computer Engineering \\ The University of British Columbia, Vancouver, Canada \\ Email: \{jinbiaox, vincentw\} @ece.ubc.ca
}

\begin{abstract}
A vehicle-to-grid (V2G) aggregator is an agent between the power grid and plug-in hybrid electrical vehicles (PHEVs). This paper studies the coordinated charging control of a V2G aggregator, which aims at minimizing the charging cost and reducing the power losses incurred by the fluctuating load. On one hand, a lower cost of charging gives the owners of PHEVs an incentive to cooperate. On the other hand, with an increasing popularity of PHEVs, the impact on the power grid such as power losses should be of concern to the aggregator. As an inherent property of a V2G aggregator, we enable bidirectional electric power flows between PHEVs and the power grid. Given the planned schedules of all the vehicles that are managed by an aggregator, we formulate the coordinated charging control as a dynamic programming problem. Due to the curse of dimensionality, we apply an approximate dynamic programming approach, which reduces the dimensionality of both state space and control space, to obtain the control sequences. We conduct simulations given the 24 -hour schedules of 100 vehicles. Simulation results show that coordinated charging control can reduce both the total cost of charging and power losses significantly, compared with the scheme where each vehicle starts charging as soon as it is connected to the grid.
\end{abstract}

\section{INTRODUCTION}

Smart grid technologies can help reducing $\mathrm{CO}_{2}$ emission and producing a cleaner environment. Recently, much attention in the power industry has been drawn to research on incorporating renewable energy into the power grid, and on increasing energy efficiency through demand response and smart control [1], [2]. For instance, plug-in hybrid electrical vehicles (PHEVs) have been used as a tool to shift demand within the transportation sector, from gasoline to electricity. As more and more PHEVs are being plugged into the power grid, the control or management issue may arise. To this end, a vehicle-to-grid (V2G) aggregator is required to decide the control sequences of a group of PHEVs based on technical constraints (e.g., the state of charge (SOC) of a PHEV) and specific objectives (e.g., minimizing the cost of charging).

Charging control [3]-[6] manages the charging sequences of PHEVs in real-time. By disabling charging of vehicles at peak time periods when the demand is high, charging control helps smoothing the load of the power grid. In [3], Clement et al. proposed an optimal charging control that minimizes the power losses of the distribution grid. They used stochastic programming to model the unknown household load at each grid node. By shifting the load of PHEVs to non-peak time periods of household loads, the system peak load and the power losses are reduced. In [4], Sortomme et al. formulated the PHEV charging problem as a quadratic program. They studied the relationship among power losses, load factor and load variance. The works in both [3] and [4] allow only power flows from the power grid to vehicles, while a V2G aggregator allows power flows in both directions.

With the deployment of smart grid technologies, the control and schedule of $\mathrm{V} 2 \mathrm{G}$ power flows (and thus bidirectional flows) become possible [7]. Recently, the growing body of literature [8]-[11] paid much attention to the use of PHEVs for frequency regulation and unit commitment. Saber et al. proposed the coordinated scheduling of PHEVs for unit commitment [9]. Han et al. proposed the scheduling of a V2G aggregator for frequency regulation [10]. By deciding when to charge and when to regulate, the aggregator maximizes each PHEV's compensation from frequency regulation against its charging cost. Sortomme et al. proposed smart charging algorithms to obtain an optimal operation point of the aggregate load of all PHEVs [11]. When the aggregator operates below the optimal point, it is considered as regulating up, and vice versa. However, Sortomme et al. assumed that V2G power flows and dynamic arrivals and departures of vehicles are disabled.

In this paper, we consider the coordinated charging control of a V2G aggregator. The goals are to minimize the total charging cost of all the vehicles at the aggregator, and to shape the aggregate load over time so as to reduce the resultant power losses. On one hand, a lower cost of charging gives the owners of PHEVs an incentive to cooperate. On the other hand, uncoordinated charging of PHEVs may impose great impact on the power grid, such as the decreased voltage, increased peak load and power losses. It is the aggregator's interest to reduce the resultant power losses, by coordinating a group of PHEVs with dynamic schedules. Considering the dynamics of user behavior, PHEVs can have different time of arrivals and departures. We assume that a planned schedule of each PHEV is known ahead of time.

The contributions of this paper are as follows:

- We formulate the coordinated charging control problem as a dynamic program. The model captures the tradeoff 


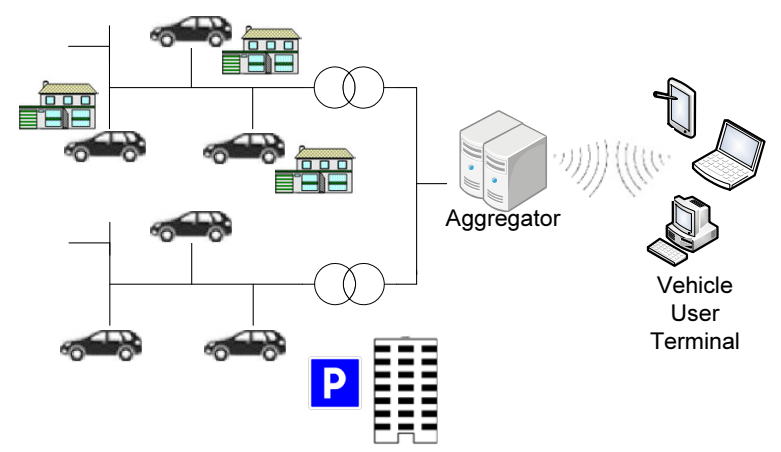

Fig. 1. The system model of a V2G aggregator.

between the total cost of charging, the power losses, and the departure penalty.

- We apply approximate dynamic programming [12], a state-of-the-art approach that tackles the curse of dimensionality [13], to solve the problem.

- We propose a coordinated charging control algorithm at a V2G aggregator.

- Simulation results show that the coordinated charging control can reduce both the total cost of charging and power losses significantly, compared with uncoordinated charging.

- The coordinated charging control with bidirectional power flows incurs much less power losses, when its total cost of charging is the same as that of the counterpart scheme with unidirectional power flows (i.e., V2G flows disabled).

Our work differs from [3] [4] in that we enable bidirectional power flows between PHEVs and the power grid whereas [3] [4] disabled the V2G power flows in order to reduce the problem size and the number of constraints. We also take into account the the dynamics of PHEVs. In our formulation, PHEVs can have different time of arrivals and departures.

This paper is organized as follows. Section II presents our system model and problem formulation. Section III describes the approximate dynamic programming approach. Section IV presents the simulation results. Conclusions are given in Section V.

\section{SySTEM MODEL}

The system model of a V2G aggregator is shown in Fig. 1. The aggregator manages multiple participating sites. When a PHEV arrives at a participating site, it can be plugged into the power grid. The aggregator operates all those present PHEVs simultaneously. The scheduling period is from time slot 1 to $T$. And the length of each time slot is $\Delta$. In this paper, we assume that a full schedule of each PHEV during the scheduling period is known before time slot 1 . Since the schedules may be volatile, the owners of PHEVs can update their schedules with the aggregator via wireless networks. If the users do not comply with the known schedule, the aggregator can re-run the scheduling algorithm when the schedule is updated.

A planned schedule of a PHEV includes the following: $a$ ) the expected arrival and departing time slots (note that each

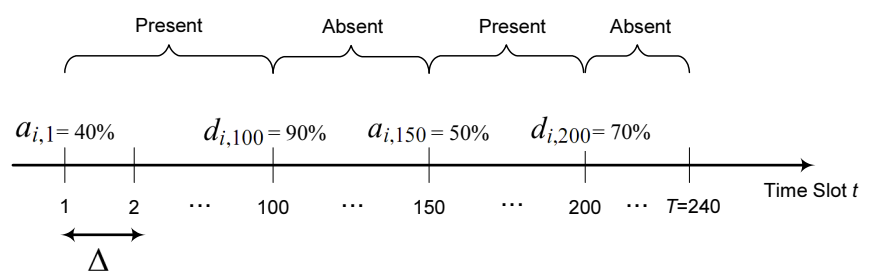

Fig. 2. An example schedule of vehicle $i$.

PHEV can arrive at and depart from the grid multiple times); $b$ ) the expected initial/target SOC at each arrival/departure time slot. Let $a_{i, t}$ denote the expected initial SOC of vehicle $i$ at arrival time slot $t$ (valid only at the arrival time slots). Similarly, $d_{i, t}$ denotes the expected target SOC of vehicle $i$ at departure time slot $t$ (valid only at the departure time slots). An example of a schedule of vehicle $i$ is shown in Fig. 2.

We assume the $\mathrm{V} 2 \mathrm{G}$ aggregator has control of a total number of $N$ vehicles. Let $s_{i, t}$ denote the state of charge (SOC) of vehicle $i$, at the beginning of time slot $t$ (we discretize $s_{i, t}$, i.e., $\left.s_{i, t} \in\{0 \%, 1 \%, \ldots, 100 \%\}\right)$. Vehicle $i$ has a maximum charging and discharging power with a magnitude of $m_{i}$. Let $\mu_{i}$ denote the number of time slots that takes vehicle $i$ to charge from empty to full capacity using full power $m_{i}$.

The normalized charging or discharging rate of vehicle $i$ at time slot $t$ is $r_{i, t}$. In this paper, $r_{i, t}$ can either take -1 (discharging at full power $m_{i}$ ), 0 (idle), or 1 (charging at full power $m_{i}$ ). We define the state variable as

$$
\mathbf{s}_{t}=\left(s_{1, t}, s_{2, t}, \ldots, s_{N, t}\right), \quad t=1, \ldots, T .
$$

The control of time slot $t \mathbf{r}_{t}=\left(r_{1, t}, r_{2, t}, \ldots, r_{N, t}\right) \in$ $U\left(\mathbf{s}_{t}\right)$, where the control space $U\left(\mathbf{s}_{t}\right)$ is

$$
\begin{aligned}
& U\left(\mathbf{s}_{t}\right) \\
& =\left\{\left(r_{1, t}, \ldots, r_{N, t}\right) \mid r_{i, t} \in\{-1,0,1\}, \forall i=1, \ldots, N\right. \text {; } \\
& r_{i, t}=0 \text {, if vehicle } i \text { is absent at time } t \text {; } \\
& 0 \% \leq\left\lfloor s_{i, t}+\frac{r_{i, t}}{\mu_{i}} 100 \%\right\rfloor \leq 100 \%,
\end{aligned}
$$

if vehicle $i$ is present in the power grid at time $t\}$.

where \lfloor\rfloor is the floor function.

Both $\mathbf{s}_{t}$ and $\mathbf{r}_{t}$ are vectors. The state transition function $f$ has the form

$$
\begin{aligned}
\mathbf{s}_{t+1} & =f\left(\mathbf{s}_{t}, \mathbf{r}_{t}\right) \\
& =\left(s_{1, t+1}, s_{2, t+1}, \ldots, s_{N, t+1}\right)
\end{aligned}
$$

For $i=1, \ldots, N$,

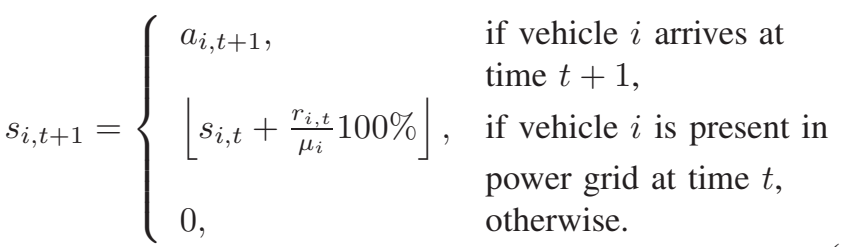

When vehicle $i$ is absent from the power grid at time slot $t$ and does not arrive at time $t+1$, we let $s_{i, t+1}=0$ by default (4). 


\section{A. Charging Cost $c_{t}\left(\mathbf{r}_{t}\right)$}

The total charging cost of the aggregator at time $t$ is

$$
c_{t}\left(\mathbf{r}_{t}\right)=p_{t} \sum_{i=1}^{N} r_{i, t} m_{i} \Delta,
$$

where $p_{t}$ is the price of electricity in time $t$. In this paper, we obtain $p_{t}$ from the day-ahead market [14]. Our formulation can be extended when $p_{t}$ is modeled as a random variable with certain probability distribution.

\section{B. Power Losses $l_{t}\left(\mathbf{r}_{t}\right)$}

The resistance of transmission lines causes the real power losses, while the reactive power losses attribute to reactive elements such as inductors. The real power losses can reduce the efficiency of transmitted energy to customers, whereas the reactive power losses may decrease the reactive power of the distribution system which is required to maintain at a certain amount for sufficient level of voltage [15]. We consider the real power losses in this paper. Let $X$ denote the number of transmission lines and $l_{t}\left(\mathbf{r}_{t}\right)$ denote the total power losses of an aggregator at time slot $t$

$$
l_{t}\left(\mathbf{r}_{t}\right)=\sum_{j=1}^{X} I_{j}^{2}\left(\mathbf{r}_{t}\right) \Omega_{j} \Delta,
$$

where $I_{j}\left(\mathbf{r}_{t}\right)$ and $\Omega_{j}$ are the current and the resistance of transmission line $j$, respectively.

In this paper, we assume the penetration level of PHEVs is $100 \%$. In other words, we consider that all the loads of the aggregator are PHEV loads. However, other loads such as random household loads can be incorporated into our problem formulation. In that case, the problem still remains as a dynamic program.

The current $I_{j}$ is the sum of power flows that pass by line $j$. Let $\Phi_{j, t}$ denote the set of vehicles, whose current passes by line $j$ in time slot $t$.

$$
I_{j}\left(\mathbf{r}_{t}\right)=\sum_{i \in \Phi_{j, t}} \frac{r_{i, t} m_{i}}{V}
$$

where $V$ is the base line voltage.

\section{Departure Penalty $g_{t}\left(\mathbf{s}_{t}\right)$}

When vehicle $i$ departs at time slot $t$ with SOC $s_{i, t}$, a penalty $g_{i, t}\left(s_{i, t}\right)$ will occur. We assume that the departure penalty is greater than zero if and only if the departure SOC $s_{i, t}$ does not reach the target SOC $d_{i, t}$. There are a variety of penalty functions $g_{i, t}\left(s_{i, t}\right)$ we can use. In this paper, we choose the quadratic function

$$
g_{i, t}\left(s_{i, t}\right)= \begin{cases}\left(d_{i, t}-s_{i, t}\right)^{2}, & \text { if vehicle } i \text { departs at } \\ 0, & \text { time } t, \text { and } s_{i, t}<d_{i, t} \\ \text { otherwise. }\end{cases}
$$

The total departure penalty of all PHEVs at time slot $t$ is

$$
g_{t}\left(\mathbf{s}_{t}\right)=\sum_{i=1}^{N} g_{i, t}\left(s_{i, t}\right) .
$$

\section{Problem Formulation}

The objective function $o_{t}\left(\mathbf{s}_{t}, \mathbf{r}_{t}\right)$ in time slot $t$ is

$$
o_{t}\left(\mathbf{s}_{t}, \mathbf{r}_{t}\right)=c_{t}\left(\mathbf{r}_{t}\right)+\lambda_{1} l_{t}\left(\mathbf{r}_{t}\right)+\lambda_{2} g_{t}\left(\mathbf{s}_{t}\right),
$$

where $\lambda_{1}$ and $\lambda_{2}$ are two positive control variables. $\lambda_{1}$ represents how many power losses (in $\mathrm{kWh}$ ) are worth $\$ 1$ charging cost. $\lambda_{2}$ represents how much departure penalty is worth $\$ 1$ charging cost.

We consider the class of policies (also referred to as control laws [12]) that consist of a sequence of sub-policies

$$
\pi=\left(\pi_{1}, \pi_{2}, \ldots, \pi_{T}\right),
$$

where $\pi_{t}$ maps state $\mathbf{s}_{t}$ into control $\mathbf{r}_{t}=\pi_{t}\left(\mathbf{s}_{t}\right)$.

We define the value functions $J_{t}^{\pi}, t=1, \ldots, T$, associated with a given policy $\pi$, as the sum of cost starting from $\mathbf{s}_{t}$.

$$
J_{t}^{\pi}\left(\mathbf{s}_{t}\right)=\sum_{k=t}^{T} o_{k}\left(\mathbf{s}_{k}, \mathbf{r}_{k}\right)=\sum_{k=t}^{T} o_{k}\left(\mathbf{s}_{k}, \pi_{k}\left(\mathbf{s}_{k}\right)\right),
$$

The optimization objective is to obtain the closed-loop optimal policy $\pi^{*}$, a feasible policy that minimizes the sum of cost starting from the initial state $\mathbf{s}_{1}$.

$$
\pi^{*}=\arg \min _{\pi} J_{1}^{\pi}\left(\mathbf{s}_{1}\right)=\arg \min _{\pi} \sum_{k=1}^{T} o_{k}\left(\mathbf{s}_{k}, \pi_{k}\left(\mathbf{s}_{k}\right)\right) .
$$

\section{Approximate Dynamic Programming}

The main issue of solving problem (13) is that both $\mathbf{s}_{t}$ and $\mathbf{r}_{t}$ are vectors. And both the state and control space will increase exponentially as the number of vehicles increases. It is also computationally intractable to enumerate all the possible states and controls at any one time slot. In this section, we use the approximate dynamic programming [12] to obtain a suboptimal solution of problem (13). State aggregation is used to reduce the state space, whereas sub-states are introduced to reduce the control space.

1) State Aggregation: We use an aggregate state space to approximate the value function of the original problem. In this paper, the aggregation function is

$$
\mathbb{H}\left(\mathbf{s}_{t}\right)=\sum_{i=1}^{N} s_{i, t} .
$$

Let $\hat{J}_{t}^{\pi^{*}}\left(\mathbb{H}\left(\mathbf{s}_{t}\right)\right)(t=1, \ldots, T)$ denote the optimal value functions of the aggregate problem. We can obtain $\hat{J}_{t}^{\pi^{*}}$ from the unique solution of Bellman's equation [13].

$$
\begin{aligned}
\hat{J}_{t}^{\pi^{*}}\left(\mathbb{H}\left(\mathbf{s}_{t}\right)\right)=\sum_{\mathbf{s} \mid \mathbb{H}(\mathbf{s})=\mathbb{H}\left(\mathbf{s}_{t}\right)} \delta_{\mathbf{s}}\left\{o_{t}\left(\mathbf{s}, \pi_{t}^{*}(\mathbf{s})\right)\right. \\
\left.+\hat{J}_{t+1}^{\pi^{*}}\left(\mathbb{H}\left(f\left(\mathbf{s}, \pi_{t}^{*}(\mathbf{s})\right)\right)\right)\right\},
\end{aligned}
$$

where $\delta_{\mathbf{s}}$ is the disaggregation probability [12]. $\delta_{\mathbf{s}}$ are the same for all s such that $\mathbb{H}(\mathbf{s})=\mathbb{H}\left(\mathbf{s}_{t}\right)$. And $\sum_{\mathbf{s} \mid \mathbb{H}(\mathbf{s})=\mathbb{H}\left(\mathbf{s}_{t}\right)} \delta_{\mathbf{s}}=1$.

Since it takes exponential time to enumerate $\forall \mathbf{s}: \mathbb{H}(\mathbf{s})=$ $\mathbb{H}\left(\mathbf{s}_{t}\right)$, we can only approximate $\hat{J}_{t}^{\pi^{*}}\left(\mathbb{H}\left(\mathbf{s}_{t}\right)\right)$ using Monte Carlo simulations. As the number of samples that we generated 
increases, the approximation for $\hat{J}_{t}^{\pi^{*}}\left(\mathbb{H}\left(\mathbf{s}_{t}\right)\right)$, denoted by $\tilde{J}_{t}^{\pi^{*}}\left(\mathbb{H}\left(\mathbf{s}_{t}\right)\right)$, will be close to optimal.

In this paper, we solve the optimal policy $\pi^{*}$ by approximating the corresponding value functions $\hat{J}_{t}^{\pi^{*}}$ by $\tilde{J}_{t}^{\pi^{*}}$, $t=1, \ldots, T$. Once we obtain $\tilde{J}_{t}^{\pi^{*}}$, the optimal policy $\pi^{*}$ can be computed as follows. For $t=1, \ldots, T$,

$$
\pi_{t}^{*}\left(\mathbf{s}_{t}\right)=\underset{\mathbf{r}_{t} \in U\left(\mathbf{s}_{t}\right)}{\arg \min }\left\{o_{t}\left(\mathbf{s}_{t}, \mathbf{r}_{t}\right)+\tilde{J}_{t+1}^{\pi^{*}}\left(\mathbb{H}\left(f\left(\mathbf{s}_{t}, \mathbf{r}_{t}\right)\right)\right)\right\} .
$$

Note that in (16), it takes exponential time to enumerate all the feasible control variables in $U\left(\mathbf{s}_{t}\right)$ since $\mathbf{r}_{t}$ is a vector. The vector space of $\mathbf{r}_{t}$ results in the curse of dimensionality [13]. To tackle this issue, we use sub-states [12] to reduce the dimensionality of the control space.

2) Sub-States: We first define the controllable set of time slot $t, \Psi_{t}$ as

$$
\begin{array}{r}
\Psi_{t}=\left\{r_{i, t} \mid \text { vehicle } i(1 \leq i \leq N)\right. \text { is present in } \\
\text { the power grid at time } t\} .
\end{array}
$$

For all vehicles that are absent at time slot $t\left(r_{i, t} \notin \Psi_{t}\right)$, we have $r_{i, t}=0$ from (2). We partition $\Psi_{t}$ equally into the sequence of $M$ groups of control variables $\Psi_{t}^{1}, \ldots, \Psi_{t}^{M}$ $\left(\Psi_{t}=\bigcup_{j=1}^{M} \Psi_{t}^{j}\right)$. Each group $\Psi_{t}^{j}(j=1, \ldots, M-1)$ contains the same number of control variables except that the last group $\Psi_{t}^{M}$ may possibly contain fewer control variables. In this paper, we let each group consist of no more than $\nu=5$ control variables. Thus, it takes at most $O\left(3^{\nu}\right)$ to enumerate all feasible controls of each group $\Psi_{t}^{j}, j=1, \ldots, M$.

We now introduce sub-states $\mathbf{s}_{t}^{1}, \mathbf{s}_{t}^{2}, \ldots, \mathbf{s}_{t}^{M}$ between $\mathbf{s}_{t}$ and $\mathbf{s}_{t+1}$. Each sub-state $\mathbf{s}_{t}^{j}(j=1, \ldots, M)$ matches with the corresponding group of control variables $\Psi_{t}^{j}$. Let $\mathbf{r}_{t}^{j}$ denote the control vector of the sub-state $\mathbf{s}_{t}^{j}$. Since $\mathbf{r}_{t}^{j}$ is associated with a group of control variables $\Psi_{t}^{j}$, we have $\mathbf{r}_{t}^{j} \in U^{j}\left(\mathbf{s}_{t}\right)$, where the control space $U^{j}\left(\mathbf{s}_{t}\right)$ is

$$
\begin{gathered}
U^{j}\left(\mathbf{s}_{t}\right) \\
=\left\{\left(r_{1, t}^{j}, \ldots, r_{N, t}^{j}\right) \mid r_{i, t}^{j} \in\{-1,0,1\}, \forall i=1, \ldots, N ;\right. \\
\quad r_{i, t}^{j}=0, \text { if } r_{i, t}^{j} \notin \Psi_{t}^{j} ; \\
\left.\quad 0 \% \leq\left\lfloor s_{i, t}+\frac{r_{i, t}^{j}}{\mu_{i}} 100 \%\right\rfloor \leq 100 \%, \text { if } r_{i, t}^{j} \in \Psi_{t}^{j}\right\} .
\end{gathered}
$$

The transition of sub-states is

$$
\mathbf{s}_{t}^{j+1}=f\left(\mathbf{s}_{t}^{j}, \mathbf{r}_{t}^{j}\right), \quad j=1, \ldots, M-1,
$$

and

$$
\mathbf{s}_{t+1}=f\left(\mathbf{s}_{t}^{M}, \mathbf{r}_{t}^{M}\right) .
$$

For $\mathbf{s}_{t}^{1}$, we have

$$
\mathbf{s}_{t}^{1}=\left(s_{1, t}^{1}, s_{2, t}^{1}, \ldots, s_{N, t}^{1}\right) .
$$

For $i=1, \ldots, N$,

$$
s_{i, t}^{1}= \begin{cases}a_{i, t+1}, & \text { if vehicle } i \text { arrives at time } t+1, \\ s_{i, t}, & \text { if vehicle } i \text { is present at time } t, \\ 0, & \text { otherwise. }\end{cases}
$$

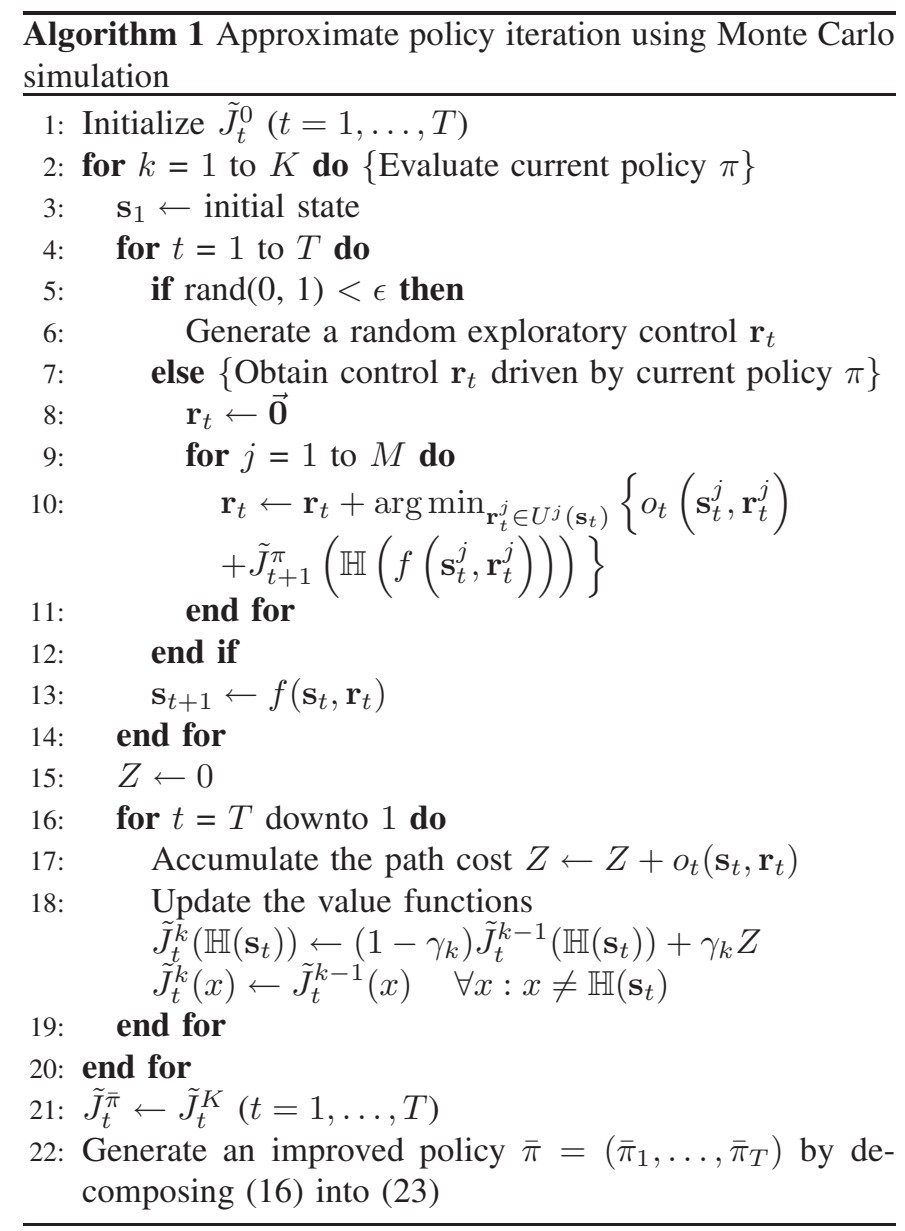

Now, we can obtain $\pi_{t}^{*}\left(\mathbf{s}_{t}\right)$ from (16) by decomposing it into a sequence of optimization problems after introducing sub-states. For $t=1, \ldots, T$ and $j=1, \ldots, M$,

$$
\pi_{t}^{* j}\left(\mathbf{s}_{t}^{j}\right)=\underset{\mathbf{r}_{t}^{j} \in U^{j}\left(\mathbf{s}_{t}\right)}{\arg \min _{t}}\left\{o_{t}\left(\mathbf{s}_{t}^{j}, \mathbf{r}_{t}^{j}\right)+\tilde{J}_{t+1}^{\pi^{*}}\left(\mathbb{H}\left(f\left(\mathbf{s}_{t}^{j}, \mathbf{r}_{t}^{j}\right)\right)\right)\right\}
$$

where $\pi_{t}^{* j}\left(\mathbf{s}_{t}^{j}\right)$ contains a valid solution for all $r_{i, t} \in \Psi_{t}^{j}$.

3) Approximate Policy Iteration Using Monte Carlo Simulation: In this section, we present a variant of approximate policy iteration [12] based on Monte Carlo simulation.

A complete policy iteration includes policy evaluation (steps $1-20$ ) and policy improvement (steps $21-22$ ) as shown in Algorithm 1. Suppose that the current policy is $\pi$. We evaluate and approximate the value functions of $\pi$ by $\tilde{J}_{t}^{k}$, $k=1, \ldots, K, t=1, \ldots, T$, using $K$ evaluation iterations. In steps $5-12$, we explore some random control with probability of $\epsilon$ (where $0<\epsilon<1$ ), otherwise we obtain the control $\mathbf{r}_{t}$ driven by current policy $\pi$. In steps $16-19$, we update the value function approximation for the aggregate state space $\tilde{J}_{t}^{k}\left(\mathbb{H}\left(\mathbf{s}_{t}\right)\right)$ with the accumulated path cost $Z$. Here, $\gamma_{k}$ is the step size for the $k$ th iteration. After we run the evaluation steps for the current policy $\pi$ by $K$ iterations, we obtain $\tilde{J}_{t}^{K}$, $t=1, \ldots, T$. Based on these approximated value functions, we generate an improved policy $\bar{\pi}$, which will be used in the next policy iteration, using the decomposition of control 


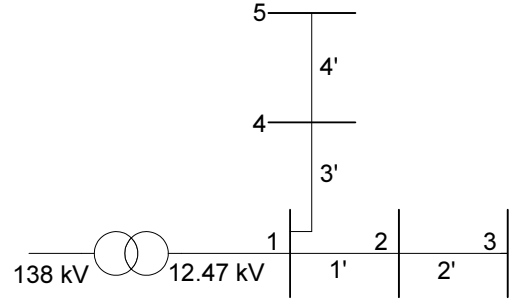

Fig. 3. One-line diagram of the 5-bus radial primary distribution system (base line voltage at $12.47 \mathrm{kV}$ ).

variables (23) with sub-states in step 22.

\section{PERFormance EVAluation}

In this section, we present numerical results of a charging control problem with a total of $N=100$ PHEVs. We consider an IEEE 5-bus radial primary distribution system in Fig. 3. Here, only buses 2 to 5 are load buses. We assign $\frac{N}{4}=25$ PHEVs equally to each load bus. There are four transmission lines $\left(1^{\prime}, 2^{\prime}, 3^{\prime}, 4^{\prime}\right)$. The resistance of each transmission line is $0.1859 \mathrm{Ohm}$. The base line voltage $V=12.47 \mathrm{kV}$.

We select $\Delta$ to be 6 minutes. The scheduling time period is from $12 \mathrm{PM}$ to the next $12 \mathrm{PM}$. Hence, $T=240$. We use the day-ahead locational marginal price (LMP) for $p_{t}$ from California ISO [14]. We assume that all $N$ vehicles have a maximum power $m_{i}=1 \mathrm{~kW}$, and their battery capacity is 10 kWh. Hence, $\mu_{i}=100$ for $i=1, \ldots, N$. In Algorithm 1, we choose $K=10^{9}$. We use a step size of $\gamma_{k}=\frac{1}{k}$ [12].

We generate the arrivals and departures of each vehicle $(i=1, \ldots, N)$ as follows. Each vehicle has two disjoint available periods that it is present in the power grid. The length of each available period is randomly distributed among $\{40, \ldots, 80\}$ time slots, namely 4 hours to 8 hours. The time slots of arrival/departure and the corresponding initial/target SOC $a_{i, t} / d_{i, t}$ are generated at random.

We first compare our proposed coordinated charging control scheme with the uncoordinated charging control scheme. The uncoordinated charging control simply let each vehicle start charging as soon as it is plugged into the grid. Each vehicle keeps charging until either the target SOC is reached or it departs from the power grid. Uncoordinated charging control is a myopic scheme that does not consider the aggregate load of all PHEVs.

Fig. 4 shows the total cost of charging of all PHEVs. The accumulated power losses of the power grid are shown in Fig. 5. As we can see, increasing the departure penalty will essentially reduce the total charging cost and accumulated power losses. However, in practice, the charging requirement of PHEVs (i.e., meeting the target SOC) should be given a high priority. In this paper, we let $\lambda_{2}=0.001,0.005$ and 0.05 . When $\lambda_{2}=0.05$, the total departure penalty of the coordinated scheme is always zero, implying that all target SOCs have been reached. Although a larger departure penalty implies further away from the target SOCs, in the worst case when $\lambda_{2}=0.001$, the average gap between the target SOC and the actual SOC, is at most $5 \%$. When $\lambda_{2}=0.005$, the average gap is at most $1 \%$.

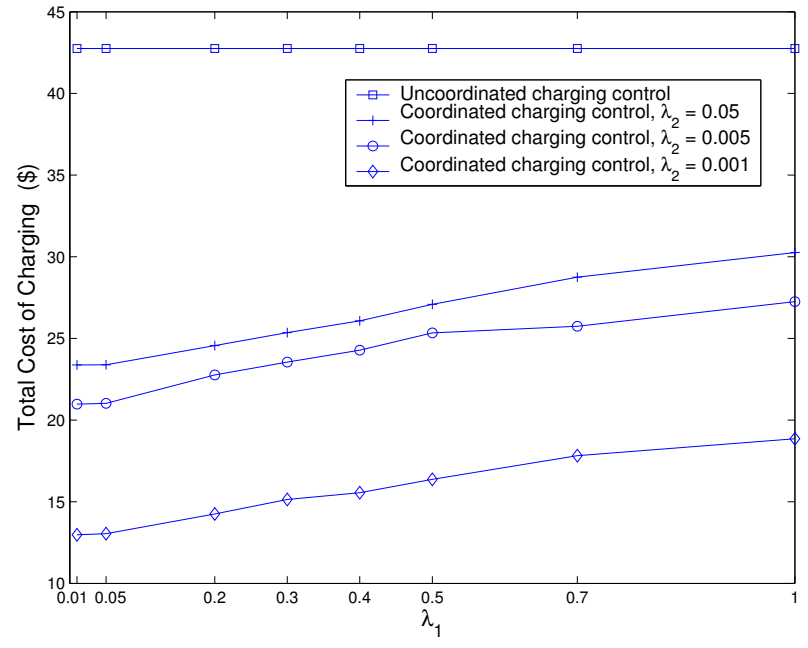

Fig. 4. The total charging cost versus the importance weight $\lambda_{1}$.

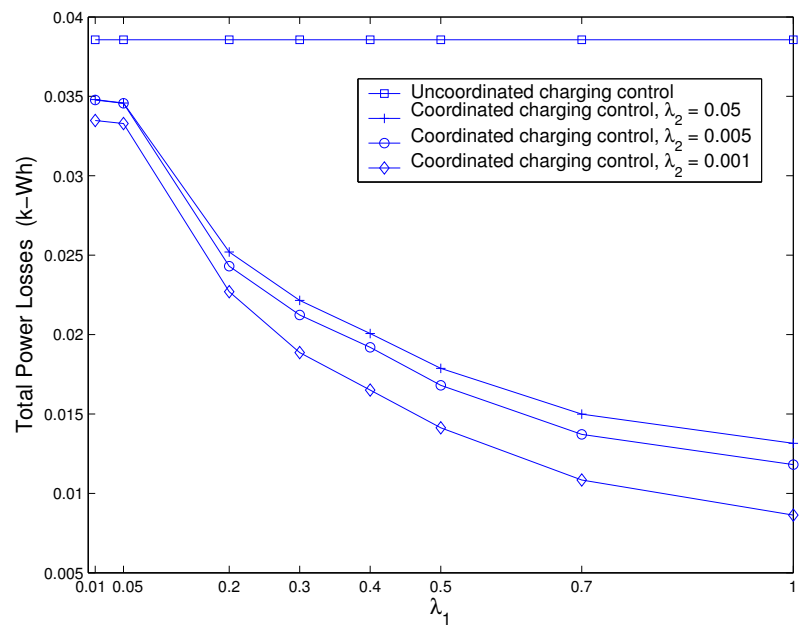

Fig. 5. The total power losses versus the importance weight $\lambda_{1}$.

Coordinated charging control has basically two advantages. First, it is price-aware. Fig. 4 shows that the coordinated scheme can reduce as much as $70 \%$ total cost of charging compared with the uncoordinated scheme when $\lambda_{1}=0.01$ and $\lambda_{2}=0.001$. Second, it enables the cooperation among different PHEVs, in order to smooth the aggregate load over time and thus reduce the power losses. Fig. 5 shows that the coordinated charging control can reduce as much as $77 \%$ accumulated power losses when $\lambda_{1}=1$ and $\lambda_{2}=0.001$. Also, results in Fig. 5 show that the total power losses decrease as $\lambda_{1}$ increases.

An interesting fact is that in Fig. 4, the lowest cost of charging is $\$ 13$ when $\lambda_{1}=0.01$ and $\lambda_{2}=0.001$, whereas the highest power losses of the coordinated scheme is 0.035 $\mathrm{kWh}$ in Fig. 5. Since the price of electricity that we used for the simulations is always less than $\$ 0.2 / \mathrm{kWh}$, the cash value of the accumulated power losses is smaller than the cost of charging. The owners of PHEVs may be reluctant to sacrifice the charging cost to reduce the power losses and thus may prefer a smaller $\lambda_{1}$. On the other hand, the power losses are concern to the V2G aggregator. As more power losses impose greater impact to the power grid, the aggregator may prefer a 


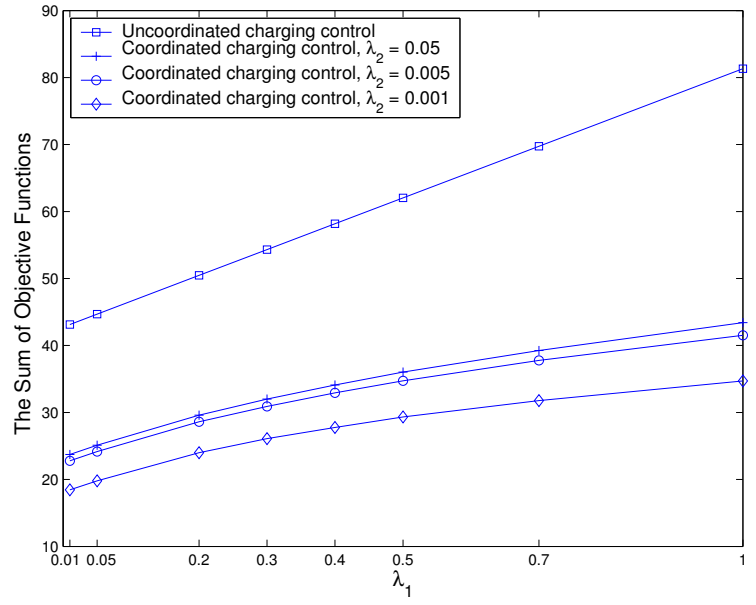

Fig. 6. The sum of objective functions from time slot 1 to $T$ versus the importance weight $\lambda_{1}$.

larger $\lambda_{1}$ in the interest of reducing the power losses.

Fig. 6 shows the sum of objective functions (i.e., eqn. (12)), starting from time 1 with initial state $\mathbf{s}_{1}$. The coordinated charging control outperforms the uncoordinated one under all scenarios we use. The reason is that the coordinated charging control reduces both the total cost of charging $\sum_{t=1}^{T} c_{t}\left(\mathbf{r}_{t}\right)$ and total power losses $\sum_{t=1}^{T} l_{t}\left(\mathbf{r}_{t}\right)$, while keeping the weighted departure penalty $\lambda_{2} \sum_{t=1}^{T} g_{t}\left(\mathbf{s}_{t}\right)$ relatively low.

To show the benefits of enabling V2G power flows, we compare the coordinated scheme with bidirectional power flows with the coordinated scheme with unidirectional flows (i.e., $r_{i, t} \in\{0,1\}$ ). For a fair comparison, we choose the total departure penalty of both schemes to be zero by setting the importance weight $\lambda_{2}$ at a large value $\left(\lambda_{2}=10\right)$. In other words, all the target SOCs in both schemes are reached. Fig. 7 shows the total cost of charging and the power losses of both schemes varying with different $\lambda_{1}$. Results show that the scheme with bidirectional flows incurs a lower power losses, when its total charging cost is the same with that of the unidirectional counterpart. Note that the coordinated scheme with unidirectional flows has a minimum cost of charging of $\$ 31.5$ when $\lambda_{1}=0$, while the one with bidirectional flows has a total cost of charging for only $\$ 27$ with the same amount of power losses where $\lambda_{1}=0.5$.

\section{CONCLUSION}

In this paper, we developed the coordinated charging control of a V2G aggregator, which reduces both the cost of charging and power losses. Given the day-ahead electricity prices and schedules of PHEVs, we formulated the problem as a dynamic program. We solved the problem using approximate dynamic programming, which reduces the dimensionality of both state and control spaces. Simulation results of an aggregator with 100 PHEVs are presented. For future work, we may further incorporate the price and schedule uncertainty into the model, making it more realistic and practical.

\section{ACKNOWLEDGMENT}

This research is supported by the Natural Sciences and Engineering Research Council (NSERC) of Canada.

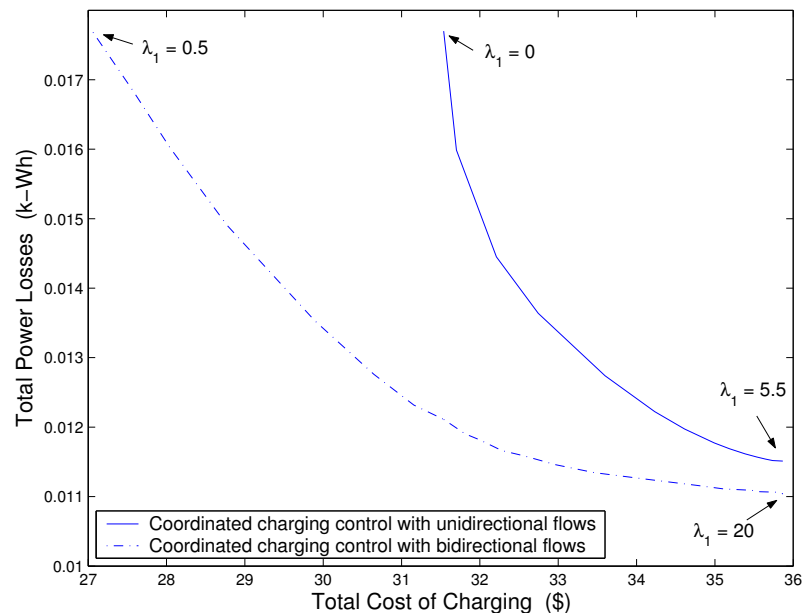

Fig. 7. Total cost of charging and power losses varying with different $\lambda_{1}$ $\left(\lambda_{2}=10\right)$.

\section{REFERENCES}

[1] A. H. Mohsenian-Rad, V. W. S. Wong, J. Jatskevich, R. Schober, and A. Leon-Garcia, "Autonomous demand side management based on game-theoretic energy consumption scheduling for the future smart grid," IEEE Trans. on Smart Grid, vol. 1, no. 3, pp. 320-331, Dec. 2010.

[2] P. Samadi, H. Mohsenian-Rad, R. Schober, V. W. S. Wong, and J. Jatskevich, "Optimal real-time pricing algorithm based on utility maximization for smart grid," in Proc. of IEEE SmartGridComm, Gaithersburg, MD, Oct. 2010.

[3] K. Clement, E. Haesen, and J. Driesen, "The impact of charging plug-in hybrid electric vehicles on a residential distribution grid," IEEE Trans. on Power Systems, vol. 25, no. 1, pp. 371-380, Feb. 2010.

[4] E. Sortomme, M. Hindi, S. MacPherson, and S. Venkata, "Coordinated charging of plug-in hybrid electric vehicles to minimize distribution system losses," IEEE Trans. on Smart Grid, vol. 2, no. 1, pp. 198 205, Mar. 2011.

[5] S. Acha, T. C. Green, and N. Shah, "Effects of optimised plug-in hybrid vehicle charging strategies on electric distribution network losses," in Proc. IEEE PES Transmission and Distribution Conf. and Exposition, New Orleans, LA, Apr. 2010.

[6] K. Parks, P. Denholm, and T. Markel, "Costs and emissions associated with plug-in hybrid electric vehicle charging in the Xcel energy Colorado service territory," Technical Report, National Renewable Energy Laboratory, May 2007.

[7] A. Ipakchi and F. Albuyeh, "Grid of the future," IEEE Power and Energy Magazine, vol. 7, no. 2, pp. 52-62, Mar. 2009.

[8] W. Kempton, V. Udo, K. Huber, K. Komara, S. Letendre, S. Baker, D. Brunner, and N. Pearre, "A test of vehicle-to-grid (V2G) for energy storage and frequency regulation in the PJM system," $U$. of Delaware, Pepco Holdings Inc., PJM and Green Mountain College, Jan. 2009.

[9] A. Saber and G. Venayagamoorthy, "Optimization of vehicle-to-grid scheduling in constrained parking lots," in Proc. IEEE PES Power and Energy Society General Meeting, New Orleans, LA, Jul. 2009.

[10] S. Han, S. Han, and K. Sezaki, "Development of an optimal vehicle-togrid aggregator for frequency regulation," IEEE Trans. on Smart Grid, vol. 1, no. 1, pp. 65-72, Jun. 2010.

[11] E. Sortomme and M. El-Sharkawi, "Optimal charging strategies for unidirectional vehicle-to-grid," IEEE Trans. on Smart Grid, vol. 2, no. 1, pp. 131-138, Mar. 2011.

[12] D. P. Bertsekas, Dynamic Programming and Optimal Control, Vol. II, 3rd ed. Athena Scientific, 2007.

[13] R. E. Bellman, Dynamic Programming, 1st ed. Princeton University Press, 1957.

[14] California ISO Open Access Same-time Information System. [Online]. Available: http://oasis.caiso.com

[15] M. Kashem, A. Le, M. Negnevitsky, and G. Ledwich, "Distributed generation for minimization of power losses in distribution systems," in Proc. IEEE Power Engineering Society General Meeting 2006, Montreal, Canada, Jun. 2006. 